\section{Results}

Of the 92 patients with gliomas $20(21.7 \%)$ had evidence of previous infection with tuberculosis as defined by the criteria above. Of the 100 controls only seven had suffered such previous infection $(7 \%)$. Previous tuberculosis was, therefore, significantly more common in the glioma cases $\left(\chi^{2}=8.613, P<0.01\right)$.

\section{Discussion}

By using the above criteria of previous tuberculous infection the data suggest that such infection is three times as common in patients with gliomas than in a random control series. This association could be explained on the basis of a common predisposing factor.

Possibly the malignancy arose as a result of increased or heightened immunological activity over a long period stimulated by and directed against the tubercle bacillus. There was, however, no clinical evidence to suggest a close temporal relation between the two conditions.

It may be that the opposite view is more tenable, that the gliomas arise-as did the tuberculosis before-in an environment favourable because of impaired immunity. The possibility that some cancers may arise where defects in immunological surveillance exist has been discussed (Lancet, 1971; Walder et al., 1971). Evidence for this has been well documented (Burnet, 1972). Firstly, cancer is more likely to arise during those periods of life when immune responsiveness is impaired-the perinatal period and old age. Secondly, there is a high risk of malignancy, particularly of intracerebral lymphomas, after immunosuppression in transplant recipients, and, thirdly, neoplasms are a common complication in certain immune deficiency states of genetic origin.

In this study it may be that patients with normal immune responses dealt rapidly with the primary infection of tuberculosis leaving no evidence of significant disease other than perhaps a healed primary focus. Those with impaired responses may have had more difficulty in dealing with the tubercle bacillus, prolonged infection being the result.

If this association between tuberculosis and cerebral gliomas is confirmed by other workers it would provide further evidence for an immunological role in the pathogenesis of neoplasia, and would call for more detailed immunological studies in patients with gliomas and tuberculosis.

We wish to thank Dr. R. R. Hughes of the Royal Southern Hospital, Liverpool, and Mr. A. Sutcliffe Kerr and his colleagues at the Regional Neurosurgical Centre, Walton Hospital, Liverpool, for permission to study their cases.

\section{References}

Burnet, M. (1972). Immunological Surveillance, Oxford, Pergamon.

Finn, R., Ward, D. W., and Mattison, M. L. (1972). British Medical Fournal, 1,111 .

Lancet, 1971, 2, 143.

Walder, B. K., Robertson, M. R., and Jeremy, D. (1971). Lancet, 2, 1282.

\title{
Subtypes of Hepatitis B Antigen in Blood Donors and Post- transfusion Hepatitis: Clinical and Epidemiological Aspects
}

\author{
STEN IWARSON, LARS MAGNIUS, ANNIKA LINDHOLM, PER LUNDIN
}

British Medical fournal, 1973, 1, 84-87

\section{Summary}

Subtyping of hepatitis B antigen (HBA) in blood donors revealed subtype ad in $56 \%$ while patients with icteric post-transfusion hepatitis from the same centre showed subtype ay in the majority of the cases $(75 \%)$. Donors with subtype ad in serum were mostly asymptomatic long-term carriers of the antigen with normal liver function ( $83 \%$ ), while $70 \%$ of donors with subtype ay in serum had signs of acute or chronic liver disease. Healthy long-term carriers of HBA seem to present little risk of transmitting hepatitis irrespective of subtype. It is, however, possible that these differences in blood donors with subtype ad and patients with post-transfusion hepatitis with subtype ay might reflect epidemiological circumstances rather than biological differences in the two viral strains.
University of Göteborg, Östra Sjukhuset, S-416 85 Göteborg, Sweden STEN IWARSON, M.D., Assistant Physician, Department of Infectious Diseases

ANNIKA LINDHOLM, M.D., Assistant Physician, Blood Centre, Sahlgren's Hospital

PER LUNDIN, M.D., Professor Pathology, Sahlgren's Hospital

Statens Bakteriologiska Laboratorium, Stockholm, Sweden

LARS MAGNIUS, M.D., Assistant Physician, Department of Virology

\section{Introduction}

The first evidence concerning heterogeneity of hepatitis B antigen (HBA) was given by Levene and Blumberg (1969), who on the basis of spur formation in gel diffusion postulated three determinants on HBA called $a, b$, and $c$. Subsequently this was confirmed by Le Bouvier (1971), who apart from a common antigenic determinant $a$ found another two determinants designated $d$ and $y$. These determinants seemed to be mutually exclusive, since hepatitis cases associated with HBA had either subtype ay or subtype ad in serum but never both. Furthermore, all cases of hepatitis B with a common source of infection carried either ad or ay. Thus it was presumed that $d$ and $y$ were two different specific strains of hepatitis B virus (Kim and Tilles 1971; Le Bouvier 1971; Mosley et al., 1972).

Other subspecificities of HBA have been described (Bancroft et al., 1972; Magnius and Espmark, 1972) but further research is needed to resolve the character and significance of these antigenic determinants.

In a previous report on blood donors (Iwarson et al., 1972) the relation between the occurrence of HBA in serum and actual signs of liver disease was described. This report presents further work on the significance of two different subtypes of HBA in serum of blood donors and includes a study of HBA subtypes in post-transfusion hepatitis cases from the same centre.

\section{Subjects}

Blood Donors.-Donors at the Blood Centre, Sahlgren's Hospital, have been tested for HBA since January 1970. The 
present study includes 32 donors with HBA in the serum discovered at routine screening of about 15,000 blood donors seen at the centre during the two-year period July 1970 to July 1972. No person with a history of hepatitis was accepted as a donor and blood samples found to contain HBA were not transfused. A detailed description of the donor population was made in a previous report (Iwarson et al., 1972). Donors found to have HBA in the serum were repeatedly examined for the antigen and also repeatedly investigated with biochemical liver function tests during a period of at least three months (range 3-20 months, mean 6 months). Serum specimens from the donors were examined for the subtypes ad and ay of HBA. During the follow-up period the donors were admitted to the infectious diseases clinic, Göteborg, for liver biopsy and additional examination of liver function.

Post-transfusion Hepatitis Cases.-This group includes 16 patients who fell ill with icteric hepatitis two to four months after having received 2-18 units of blood obtained from the Blood Centre, Sahlgren's Hospital. These hepatitis cases were reported to the centre from June 1969 to January 1972 and all were typical cases of acute icteric viral hepatitis with HBA in the serum. Acute-phase serum specimens from these presumed cases of post-transfusion hepatitis were examined for the HBA subtypes $a d$ and $a y$. During the period of study a further 17 cases of clinical hepatitis associated with blood transfusions were reported to the centre. These cases were all HBA-negative but some of them had first been tested more than two weeks after observation of jaundice. There was no systematic follow-up of blood recipients to search for subclinical cases of hepatitis.

\section{Methods}

\section{BIOCHEMICAL AND IMMUNOCHEMICAL ANALYSES}

The following biochemical liver function tests were employed: serum bilirubin, thymol turbidity, serum alkaline phosphatase, serum aspartateaminotransferase (SGOT), serum alanine aminotransferase (SGPT), and galactose tolerance. The performance of these tests and the normal criteria used were described previously (Iwarson and Hermodsson, 1971). HBA was determined by immunodiffusion during the first 6 months of the study period and after that in addition by counter-electrophoresis. The detailed performances of these methods have also been previously described (Iwarson et al., 1972). Subtyping of HBA was performed as described in a previous report with a few modifications (Magnius and Espmark, 1972). Three of the four antisera used, $A b$ 11, $\mathrm{Ab}$ 34, and B.A., were also characterized in that report.

Rabbit anti- $d$ was obtained after immunization with a partially purified ad antigen. The purification procedure involved the following steps. Serum $(70 \mathrm{ml})$ from a persistent carrier of HBA of the subtype ad was precipitated with $13 \%$ polyethyleneglycol (Carbowax 6,000). The precipitate was dissolved in $0.025 \mathrm{M}$ sodium acetate buffer at $\mathrm{pH} 5 \cdot 8$. A 4-ml sample of the supernatant was subjected to zonal centrifugation in a $60 \mathrm{ml}$ preformed sucrose gradient ranging from $5 \%$ to $20 \%$ sucrose in purified buffered saline with an SW $25: 2$ rotor in a Beckman G ultracentrifuge (Spinco Division, California) at 15,000 r.p.m. and $4^{\circ} \mathrm{C}$ for 66 hours. A total of $7.5 \mathrm{ml}$ consisting of the bottom fractions of the sucrose gradient and containing the HBA was layered on $16.5 \mathrm{ml} \mathrm{CsCl}$ in purified buffered saline of S.G. $1 \cdot 880$. Purified buffered saline was added to a final volume of $60 \mathrm{ml}$ and the material was subjected to isopycnic banding in the same rotor and centrifuged at 22,500 r.p.m. and $4^{\circ} \mathrm{C}$ for 160 hours. Five rabbits were injected three times weekly by the subcutaneous route with $0.1 \mathrm{ml}$ of material obtained from the HBA peak emulsified in $0.1 \mathrm{ml}$ Bacto Complete Freund Adjuvant (Difco Laboratories, Detroit, Michigan) and were bled 10 days after the last injection. One rabbit serum showed a strong antibody reaction against HBA in immuno-diffusion and could be used as an anti-d after in-well absorption with ay antigen from a patient undergoing maintenance haemodialysis treatment.
HISTOPATHOLOGICAL ANALYSES

Liver biopsy was performed through a transthoracic route according to Lundvall and Iwarson (1970). The histopathological classification of acute viral hepatitis, chronic persistent hepatitis, and unspecified reactive hepatitis was made according to principles given by international groups (Groote et al., 1968; Bianchi et al., 1971).

\section{Results}

\section{DISTRIBUTION OF HBA SUBTYPES}

The frequency of the two subtypes ad and ay in blood donors and post-transfusion hepatitis cases from Sahlgren's Hospital, Göteborg, is shown in table I. The blood donors showed subtype ad in $56 \%$, while most of the post-transfusion hepatitis cases $(75 \%)$ were found to have subtype ay in the serum.

TABLE I-HBA Subtypes ay and ad in Blood Donors and Post-transfusion Hepatitis Cases

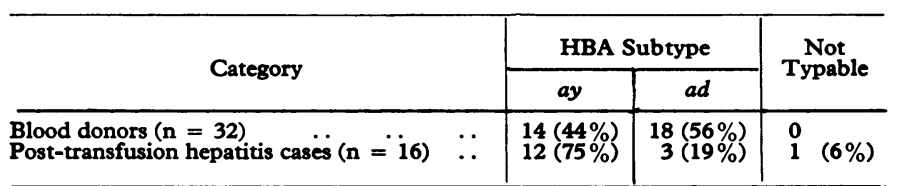

\section{SUBTYPES AND LIVER DISEASE IN DONORS}

Seven donors $(22 \%)$ developed clinical hepatitis with jaundice two to four months after HBA was discovered in the serum at routine screening (table II). Liver biopsy was performed during the acute illness in three of the cases and showed the classical appearance of acute viral hepatitis according to Baggenstoss (1957). The disease also had the typical course with liver function returning to normal within three months. HBA was only transiently demonstrable (three to six weeks) in this group, which included six donors with subtype ay and one donor with subtype ad in the serum. Three of the donors with subtype ay were found to be drug addicts.

TABLE II-HBA Subtypes and Liver Function in 32 Swedish Blood Donors with this Antigen in Serum

\begin{tabular}{|c|c|c|c|c|c|}
\hline Category of Donors & & $\begin{array}{l}\text { No. with } \\
\text { Acute } \\
\text { Liver Dys- } \\
\text { function }\end{array}$ & $\begin{array}{l}\text { No. with } \\
\text { Chronic } \\
\text { Liver Dys- } \\
\text { function }\end{array}$ & $\begin{array}{l}\text { No. with } \\
\text { Normal } \\
\text { Liver } \\
\text { Function }\end{array}$ & $\underset{\text { Followed }}{\text { Not }}$ \\
\hline $\begin{array}{l}\text { HBA subtype ay in serum } \\
\text { (n = 14) } \\
\text { HBA subtype ad in serum } \\
\begin{array}{c}(\mathrm{n}=18) \\
\text { (1) }\end{array}\end{array}$ & $\begin{array}{l}. \\
\cdots \\
\cdots\end{array}$ & $\begin{array}{l}6(49 \%) \\
1 \quad(6 \%)\end{array}$ & $\begin{array}{l}4(29 \%) \\
1 \quad(6 \%)\end{array}$ & $\begin{array}{r}3(21 \%) \\
16(88 \%)\end{array}$ & $\begin{array}{ll}1 & (7 \%) \\
0 & \end{array}$ \\
\hline Total & $\ldots$ & $7(22 \%)$ & $5(16 \%)$ & $19(59 \%)$ & $1(3 \%)$ \\
\hline
\end{tabular}

Raised liver transaminases (SGPT, SGOT) during the entire follow-up period (3-20 months) was noted in five donors (16\%), and four of them had subtype ay in the serum (table II). Liver biopsy specimens showed changes consistent with those of chronic persistent hepatitis in four cases, including the one with subtype $a d$, while the remaining donor had histological alterations classified as non-specific reactive hepatitis. All five donors within this group were carriers of HBA during the entire followup period. Three of them, all with chronic persistent hepatitis and subtype ay in the serum, were found to be drug addicts.

Normal liver function was noted in 19 donors (59\%). Three of them had subtype ay in the serum, while 16 showed subtype ad (table II). Liver biopsy showed normal liver structure in 10 cases (including one donor with subtype ay), while minor histological changes such as slight steatosis (three cases) or unspecific reactive hepatitis (five cases) were noted in the remaining cases. In one donor of this group liver biopsy was not performed. 
RELATION BETWEEN HBA SUBTYPE IN DONORS AND CLINICAL HEPATITIS IN RFCIPIENTS

Of the 32 donors with HBA in the serum five had been involved in a known case of icteric post-transfusion hepatitis (table III).

TABLE III-Relation between Transfusions of presumably $H B A$-positive Blood Units of Either Subtype and Development of Clinical Post-transfusion Hepatitis

\begin{tabular}{|c|c|c|c|c|c|c|}
\hline & & & & $\begin{array}{l}\text { No. of } \\
\text { Donors } \\
\text { Involved }\end{array}$ & $\begin{array}{l}\text { No. of } \\
\text { Blood } \\
\text { Units } \\
\text { Transfused }\end{array}$ & $\begin{array}{l}\text { Reported } \\
\text { Cases of } \\
\text { Clinical } \\
\text { Hepatitis }\end{array}$ \\
\hline $\begin{array}{l}\text { Subtype ay } \\
\text { Subtype ad }\end{array}$ & $\because$ & $\because$ & $\begin{array}{l}\text { Long } \\
\ldots \\
\cdots\end{array}$ & $\begin{array}{c}\text { Serm Carriers } \\
4 \\
17\end{array}$ & \begin{tabular}{|r}
$B A$ \\
30 \\
275
\end{tabular} & $\begin{array}{l}1(3.3 \%) \\
2(0.7 \%)\end{array}$ \\
\hline Subtype ay & $\ldots$ & $\begin{array}{c}\text { Hepati } \\
\ldots\end{array}$ & s-incut & ${ }_{2}$ & irriers of $H B A$ & 2 \\
\hline
\end{tabular}

The 16 long-term carriers of subtype ad with normal liver function had donated a total of 253 units of blood during the years before testing for HBA was started. Only two of these donors, who had donated 40 and 59 units respectively, had been involved in a case of known clinical post-transfusion hepatitis. The presumed long-term carriers of subtype ay without signs of liver disease (three donors) had together donated 24 units of blood before they were excluded. None of these donors had been involved in a known case of post-transfusion hepatitis.

Two of the donors who were found to have chronic persistent hepatitis and were long-term carriers of HBA in the serum, had donated blood before testing for the antigen was started. One of them, who showed subtype ad in serum, had donated 22 units of blood and had not been involved in any reported case of posttransfusion hepatitis. The other one, who was a drug addict and showed subtype ay in the serum, had donated six units. This donor was involved in a case of icteric post-transfusion hepatitis about 10 months before he was first tested for HBA and excluded from the donor population.

Two of the donors, who subsequently developed clinical hepatitis, both with subtype ay in the serum, had donated one blood unit each previously during the incubation period of the disease. These units were found to be HBA-negative by immunodiffusion and were transfused. However, clinical hepatitis developed in both the recipients of these blood units.

\section{Discussion}

Preliminary reports indicate that subtype ay of $\mathrm{HBA}$ is more likely to occur in clinical hepatitis, while subtype ad is more often found in chronic asymptomatic carriers of the antigen (Holland et al., 1972; Magnius et al., 1972a). The present study of blood donors and post-transfusion hepatitis cases largely confirms these observations. The clinical post-transfusion hepatitis cases showed subtype ay in $75 \%$. Six out of seven donors, who developed icteric viral hepatitis, had HBA subtype ay in serum, while 16 out of 19 asymptomatic presumed long-term carriers of HBA with normal liver function showed subtype $a d$ in the serum. The hepatitis cases had demonstrable antigen in serum for only a few weeks, while the asymptomatic donors with normal liver function were long-term carriers of the antigen - that is, HBA was detectable in the serum for more than three months (Krugman and Giles, 1970).

It has also been proposed that subtype ad is more often found in chronic hepatitis (Holland et al., 1972). In the present study four out of five donors with histopathologically verified chronic persistent hepatitis showed HBA subtype ay in the serum. However, three of these donors were drug addicts and it is possible that chronic persistent hepatitis in drug addicts is more often associated with HBA subtype ay. A great prevalence for this subtype has been reported in outbreaks of acute viral hepatitis among drug addicts (Schmidt et al., 1972).

In some of the asymptomatic carriers of HBA with normal liver function discreet histopathological alterations consistent with those of unspecific reactive hepatitis (five cases) and slight steatosis (three cases) were noted. These alterations might represent sequelae after a subclinical form of viral hepatitis but might as well be quite unspecific.

In the present donor population HBA subtype ay was noted in high frequency $(44 \%)$ as compared with other observations (Holland et al., 1972; Magnius et al., 1972a), which indicated a rather low incidence of subtype ay in donor populations (6-15\%). One reason for this difference might be that six of the donors in the ay group $(43 \%)$ were drug addicts. Another possible explanation might be that as many as seven donors were picked up in the incubation period of clinical hepatitis. In Sweden most clinical hepatitis cases are associated with HBA subtype ay (Magnius et al., 1972a). When the donors who developed clinical hepatitis and the donors found to be drug addicts are excluded from the present material the remaining donors show subtype ay in only $23 \%$ of the cases. The distribution of HBA subtypes obtained in this reduced material is probably more comparable with the results obtained in other donor populations.

The clinical and histopathological differences observed in individuals with subtype ad and ay respectively might reflect differences in viral strains. However, it seems more reasonable to postulate that these differences are attributed to epidemiological circumstances. The intense liver injury in clinical hepatitis seems to be associated with a short period of antigenaemia, while a subclinical infection is more apt to be associated with a long period of antigenaemia (Iwarson et al., 1972). In Sweden there is some evidence for a change in the dominant subtype of HBA associated with clinical hepatitis. As will be reported elsewhere (Magnius et al., 1972b) sera from patients with overt, clinical viral hepatitis, collected in Stockholm in 1953, showed predominantly subtype $a d$, while today subtype ay is dominant within the same area. Provided there has been a change in the predominating subtype and there is a relation between subclinical infection and long-term antigenaemia it is not surprising to find subtype ad in most asymptomatic longterm carriers of HBA among blood donors without clinical hepatitis in the history.

Surprisingly the long-term carriers with normal liver function showed about the same involvement in reported post-transfusion hepatitis cases as did donors without demonstrable HBA in the serum - that is, only two out of 20 donors had been involved in a reported case of post-transfusion hepatitis (and then in 1963 and 1964 respectively). However, nothing is known about involvement in subclinical post-transfusion hepatitis cases, which probably are more prevalent (Cherubin, 1971). Blood obtained from two donors in the incubation period of viral hepatitis was associated with clinical hepatitis in both recipients. This finding together with the observation of a high ay rate in the present series of post-transfusion hepatitis, which probably reflects the predominance of this subtype among clinical hepatitis cases in Sweden to-day, certainly stress the importance of avoiding blood obtained from donors in the incubation period of viral hepatitis. This can at present be done only by testing each unit of blood for HBA with a rapid and sensitive method before transfusion.

Infectivity of blood containing HBA of either subtype cannot with certainty be equated with infectivity of the corresponding viral strain. Certainly differences in infectivity of HBA-positive sera do not reflect differences in strain virulence if the positive sera of one subtype come from individuals in the incubation period of viral hepatitis and positive sera of the other subtype are derived from persistent carriers of the antigen. As is the case with other viral diseases the infectious agent is presumably most prominent in serum of individuals during the incubation period or the early stage of the illness.

In conclusion, the different distribution of the subtypes ad and $a y$ in blood donors and in post-transfusion hepatitis cases might reflect epidemiological circumstances rather than biological differences in the corresponding viral strains. Longterm carriers of HBA with normal liver function seem to present little risk of being contagious, while it seems most important to avoid donors who are in the incubation period of a clinical or 
subclinical viral hepatitis. Consequently there should be little risk in otherwise healthy long-term carriers of HBA visiting barbers or dentists or working as nurses, physicians, and so on. Earlier opinions on this problem (Chalmers and Alter, 1971) can probably be modified provided that the results of this study are confirmed.

\section{References}

Baggenstoss, A. H. (1957). Fournal of the 'American Medical Association'

Bancroft, W. H., Mundon, F. K., and Rusell, P. K. (1972). Fournal of Immunology, 109, 842 .

Bianchi, L., et al. (1971). Lancet, 1, 333

Chalmers, T. C., and Alter, H. J. (1971). New England fournal of Medicine, $285,613$.

Cherubin, C. E. (1971). Lancet, 1, 627.

Groote, J. de, et al. (1968). Lancet, $2,626$.
Holland, P. V., Purcell, R. H., Smith, H., and Alter, H. J. (1972). Hepatitis Scientific Memoranda (Memo H-283), NIH, USA

Iwarson, S., and Hermodsson, S. (1971). Scandinavian fournal of Infectious Diseases, 3, 93.

Iwarson, S., Lindholm, A., Lundin, P., and Hermodsson, S. (1972). Vox Sanguinis, 22, 501.

Kim, C. Y., and Tilles, J. G. (1971). Fournal of Infectious Diseases, 123, 618 Krugman, S., and Giles, J. P. (1970). Fournal of the American Medical Association, 212, 1019.

Le Bouvier (1971). Fournal of Infectious Diseases, 123, 671.

Lundvall, O., and Iwarson, S. (1970). Acta Medica Scandinavica, 187, 225.

Levene, C., and Blumberg, B. S. (1969). Nature, 221, 195.

Levene, C., and Blumberg, B. S. (1969). Nature, 221, 195.

Magnius, L.-O., and Espmark, A. (1972). Fournal of Immunology, 109, 1017

Microbiologica Scandinavica, 80, 340.

Magnius, L.-O., Berg, R. Bjorvatn, B., Espmark, J. Å., and Svedmyr, A.

Mosley, J. W., Edwards, W. M., Neikans, J. E., and Reidecher, A. G. (1972). American fournal of Epidemiology, 95, 529.

Schmidt, N. J., Roberto, R. R., and Lennette, E. H. (1972). Infection and Immunity, 6, 1.

\section{MEDICAL MEMORANDA}

\section{Congenital Hemihypertrophy with Aortic, Skeletal, and Ocular Abnormalities}

\section{HENRY, J. P. LOUIS, J. C. HOEFFEL, C. PERNOT}

British Medical fournal, 1973, 1, 87-88

Congenital hemihypertrophy is a rare malformation in which one half of the body is more developed than the other.

\section{Case Report}

A woman aged 24 was found on routine premarital examination to have a cardiac murmur and anomalies of the limb extremities. There was no history of previous illness although she walked with a slight limp.

She was admitted to hospital for investigation. On examination there was striking asymmetry of the body (fig. 1), particularly the upper part. The right upper limb was $4 \mathrm{~cm}$ longer than the left and was thicker. The difference was less noticeable in the lower limbs, the right leg being $3 \mathrm{~cm}$ longer than the left. The face showed a mild asymmetry; the right eye was higher than the left, the right ear larger, the right part of the lip thicker, and the chin more prominent on the right side. The teeth and tongue were symmetrical. The left iris was more deeply coloured than the right. Several fingers of the left hand were shortened. There was deformity of the big toes together with syndactyly of the second and third toes on each side. The right breast was larger than the left. Radiographs of the skeleton showed that on the right side each bone was thicker and longer than its counterpart on the left. In addition, the right femoral head was incompletely covered by the acetabulum. Films of the hands (fig. 2) showed, on the left side, shortening of the first metacarpal and first phalanx of the thumb and clinodactyly of the first and fifth fingers. The cubital styloid was absent. Radiographs of the feet (fig. 3) showed that on the right side there was clinodactyly of the second phalanx of the great toe, absence of the second phalanx of the fifth toe, and shortening of the second phalanx of the other toes. On the left foot there was shortening of the metatarsal bones and of the first phalanges.

Blood pressure was $150 / 70 \mathrm{~mm} \mathrm{Hg}$ in the right arm and $110 / 70 \mathrm{~mm} \mathrm{Hg}$ in the left. There was a rough systolic murmur situated high up in the aortic area below the clavicle. It was of medium intensity and radiated into the neck. The second sound

University Hospital Jeanne d'Arc, 54-Dommartin-les-Toul, France M. HENRY, M.D., Cardiologist, Assistant Chef de Clinique J. P. LOUIS, M.D., Radiologist

J. C. HOEFFEL, M.D., Professor of Radiology, Head of Radiology Department

C. PERNOT, M.D., Professor of Cardiology, Head of Department of Medicine and Cardiology

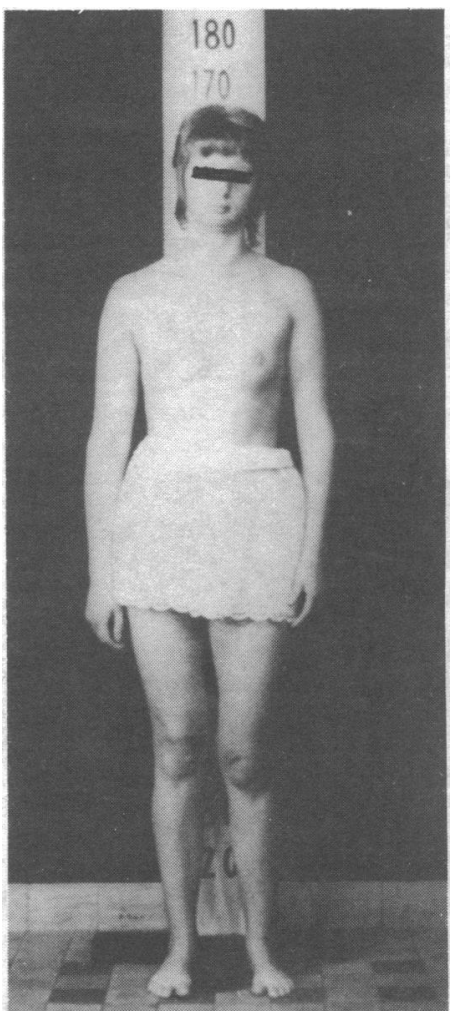

Mg 1-Patient, showing rightsided hemihypertrophy.

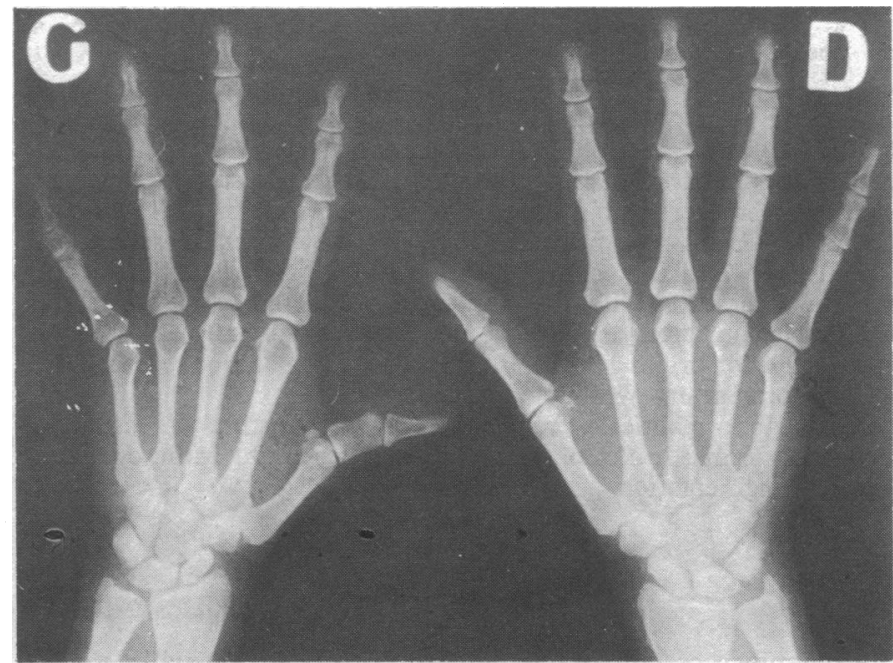

FIG. 2-Radiographs of hands. 